The effect of average storage temperature, and temperature fluctuation on the rate of moisture migration in a model frozen food

\author{
Reid, D.S. and, Perez Albela, L \\ Food Science and Technology \\ University of California, Davis \\ Davis, CA, 95616, USA \\ Emaildsreid@ucdavis.edu
}

\begin{abstract}
When frozen food is stored for an extended period, signs of moisture migration are frequently evident. In this study a model frozen food (frozen starch gel cylinders) has been stored at average temperatures of $-12,-15,-18$, and $-20^{\circ} \mathrm{C}$. These temperatures are the result of cycling storage at temperatures either 5 or 10 degrees apart. Hence both the effect of average temperature and the effect of the size of the temperature fluctuation can be studied. The rate of moisture migration was quantified by regular weighing (in the cold room) of each individual frozen gel. In this way problems due to moisture pick-up through condensation were minimized. The results clearly confirmed prior observations that the rates of moisture migration were increased at the higher average temperatures of storage. In addition, it could be clearly seen that the rate of moisture migration was increased for larger amplitude temperature fluctuation. This result differs from the observation that only the average temperature, and not the magnitude of temperature fluctuation is significant for the rate of chemical change in frozen storage. It is a clear indicator that both the average temperature, and the magnitude of temperature fluctuation are important when considering the rates of physical processes during frozen storage. The sensitivity of the process to temperature fluctuation is reduced at the lower average temperatures.
\end{abstract}

Keywords: frozen storage, temperature fluctuation, moisture migration

\title{
Justification for study
}


The purpose of freezer storage is to obtain extended high quality shelf life. The maintenance of storage temperature is important. Temperature fluctuations influence quality factors such as ice crystal size, and moisture migration differently from quality factors such as flavor, color etc. Ice crystal size distribution and moisture migration are factors uniquely sensitive to temperature fluctuations, and to the establishment of temperature gradients within a frozen stored product. Given the current energy crisis both energy conservation, and energy cost management have become major issues to the warehouseman. The question to be addressed is that of the sensitivity of product quality to various power-use management, and power-cost management strategies that may be designed and implemented. This is not an easy question to address. The sensitivity of a product to a fluctuating temperature environment depends upon many factors.

These factors include

- The size of the thermal unit (i.e. the "block" which is in contact with the air of the storage room.

- The construction of the block (i.e. is it a tote, a case, a palette of cases, etc)

- The internal thermal properties of this block (i.e. the ease of transfer of heat within the case, carton, package or palette.)

- The existence and location of moisture transfer barriers within the block. These barriers define the "unit barrier package".

- The effectiveness of thermal contact between the cold-room air and the surfaces of the block, under active cooling, and under "shut-down"

- The range of the temperature fluctuations of the external cold-room air.

- The time pattern of these temperature fluctuations.

- The refrigeration capacity of the room when cooling is on.

As an illustration of the interplay of the factors, consider a palette, shrink- wrapped, made up of cases of product. The thermal properties are that of the palette. Heat transfer from the room air is across the shrink-wrap. The efficiency of heat transfer depends upon air temperature, and air velocity at the surface. Within the palette, heat transfer is governed by thermal conductivity. Each case can be considered as a unit, 
and the palette is an assembly of units. However, each case internally is an assemblage of product packages. Each package has its own thermal character, and the case properties are a summation of the properties of the packages. Given the appropriate information, it is possible to model the heat transfer behavior of the palette, and predict the change in temperature of any particular case, and also the changes in temperature of the packages within the case. The predictions can readily be confirmed by appropriate measurements. What is less well known are the drivers for moisture movement. Moisture barriers interrupt moisture transfer. Two thermally identical palettes can differ significantly in the effectiveness, and location, of moisture barriers, so that even though the overall internal thermal history of the palettes could be identical, the patterns of moisture transfer could be very different. In other words, though identical as thermal units their internal structures are described as assemblies of different "unit barrier packages" which, however, result in the same overall thermal assembly.

Essentially, quality issues are governed by the temperature fluctuations exhibited in the "unit barrier package", whatever that may be, while the extent of fluctuation at any spatial location within the thermal unit is governed by the relationship between the thermal unit and the cooling capability of the storage room.

Where critical quality loss is due to chemical changes, the USDA studies of the 1950s (Van Arsdel, Copley et al. 1969) showed that the rate of change in most cases is adequately approximated by using the deterioration rate which applies at the average temperature of the storage unit (i.e. product package). Where critical quality changes are linked to physical processes, such as ice recrystallization and moisture migration, the size of the temperature fluctuation is always of concern, as well as the average temperature. Larger fluctuations lead to more rapid change. For example, Ice crystal size changes more rapidly for a \pm 1 degree change at $20^{\circ} \mathrm{F}$ than for $\mathrm{a} \pm 1$ degree change at $10^{\circ} \mathrm{F}$. At either average temperature, they change more rapidly for a \pm 2 degree change compared to $\mathrm{a} \pm 1$ degree change

For moisture migration, the fluctuations in external temperature lead to the establishment of internal temperature gradients, which also change with time. 
Moisture migration is driven by temperature gradients. The steeper the temperature gradient, the more rapid the transfer of moisture. As with the transfer of moisture to the freezer coils (being the coldest part of the system), within the "unit barrier package", which may be a retail package, or may be a tote, moisture travels toward the lowest temperature point. Temperature fluctuation produces complex, ever changing temperature gradients. Individual situations need to be analyzed to determine the maximum fluctuation allowable in the temperature of the external coldroom atmosphere before temperature gradients are set up within the stored product that lead to an accelerated process of moisture migration. A critical threshold for significant change must be established. There are few extant studied of moisture migration (Moleeratanond, Kramer et al. 1979)

The new realities of energy supply and demand, together with our increased understanding of the sensitivity of products to fluctuating temperature make it important that we re-examine the costs and benefits of energy management schemes. In particular, it is important that we identify those products that may be uniquely at risk to fluctuations in temperature consequential on various load-management schemes.

\section{Experimental design}

The purpose of this project was to determine what level of risk, if any, to product quality is associated with the use of power load management schemes to optimize energy usage costs. The procedure was to determine the level of risk associated with physical processes of deterioration. To address this objective, this project aimed to determine the effect on moisture migration/redistribution in $10 \%$ gelatinized starch gels under constant temperature and fluctuating averaged-equivalent temperature, and to determine the effect on the amplitude of temperature fluctuation on the redistribution of moisture $\left(5 \mathrm{vs} .10^{\circ} \mathrm{C}\right)$.

The temperature conditions employed throughout the study were:

$-12^{\circ} \mathrm{C}$ vs. -10 to $-15^{\circ} \mathrm{C}$

$-15^{\circ} \mathrm{C}$ vs. -10 to -20 and -12 to $-17^{\circ} \mathrm{C}$ 
$-17^{\circ} \mathrm{C}$ vs. -12 to -22 and -15 to $-20^{\circ} \mathrm{C}$

$-20^{\circ} \mathrm{C}$ vs. -17 to $-22^{\circ} \mathrm{C}$

These temperatures were maintained in a suite of specially modified chest freezers, each with precise thermostatic control of temperature, and internal fan movement of air to reduce temperature fluctuations.

Samples were stored at either at constant temperatures in a single freezer or moved between two freezers set at the limit temperatures described above on a 12 hour cycle, thus establishing both the amplitude of the fluctuation, and the average storage temperature.

\section{Moisture redistribution:}

Moisture redistribution during storage leads to weight loss, freezer burn, ice crystals on the surface of products etc. This is a physical change which results from diffusion driven by temperature gradients, and their accompanying water vapor pressure gradients. Diffusion is from high to low temperature. Diffusion to the exterior is easier than the reverse diffusion. We chose to study migration by determining weight loss during storage, and also to make all determinations at sub-freezing temperature to minimize the effect of temperature fluctuations associated with the measuring step, and weight gains due to condensation of moisture on the samples.

\section{Materials and methods:}

A $10 \%$ starch solution was prepared and heated until gelatinized. While still hot it was poured into a series of 1 " by 5 " tubes, cooled and frozen. The frozen starch rods were removed and placed in holding baskets, contained in sealed Ziploc bags. 16 starch gels were placed in each basket in a 4 columns and 4 rows distribution, and the basket was then placed in the Ziploc bag.. The evolution of the weight of the 12 individual outer gels (outer layer) and 4 inner gels (inner layer) in each basket was monitored over time. Each rod could be identified, and its individual weight profile monitored. The change in weight (and amount of frost) was determined by scraping 
off from the rod prior to reweighing the rod. It was confirmed that the total amount of frost was matched by the total weight loss from rods. The frost was retained in the ziplock bag throughout the experiment, in order to determine whether there was any weight loss due to diffusion through the bag. . From the individual weight histories of rods, and the amount of frost produced it could be confirmed that there was no weight loss from the basket/bag combination during 35 weeks of storage. All weighings were performed on a balance held in $\mathrm{a}-10^{\circ} \mathrm{C}$ room to minimize artifacts caused by condensation effects on the rods. Removal of the rod from its location in the rack, and weighing, and return to the rack was performed rapidly to minimize temperature fluctuation due to the procedure. Each bag was handled individually, and returned to storage in the minimum time possible

\section{Results:}

The results of moisture redistribution over a 35 week storage period are shown in Figures 1 through 5. Figure 1 shows the average cumulative weight loss for all four storage temperatures. Figure 2 compares weight loss for the inner and outer rod layers.. Figure 3 shows the weight loss for different amplitude oscillations at an average of $-20^{\circ} \mathrm{C}$. Figure 4 shows the result for an average temperature of $-17^{\circ \mathrm{C}} \mathrm{C}$. Figure 5 shows the effect of a $5^{\circ}$ oscillation at the four average temperatures. The results show that there is a greater weight loss at the higher storage temperatures, and also for the inner layer of rods that the outer layer rods. The weight loss is higher for oscillating temperature conditions than for constant temperature conditions and the greater the amplitude of the temperature fluctuation, the greater is the extent of moisture redistribution when compared to constant temperature storage. Data collected at intermediate storage times also show that the longer the storage time, the greater the weight loss, and the greater the difference in weight loss between the inner and outer layers..

No significant variation in the total weight of the samples was observed (data not shown), this indicates that the amount of moisture that migrated out through the 
Ziplocs bags is negligible, indicating a steady state of the distribution of the moisture in the amount of frost, inner and outer layer weight loss.

\section{Conclusion}

These results clearly show that the amplitude of temperature fluctuation, as well as the average temperature maintained, both influence the extent of moisture migration. To minimize migration, the temperature should be as low as possible. The influence of temperature fluctuation is increasingly important as the average temperature of storage rises. Hence it is important to control not only the average temperature of storage, but also the extent of fluctuation. Fluctuations have an acceptably small effect providing that the storage temperature is sufficiently low. 
Figures

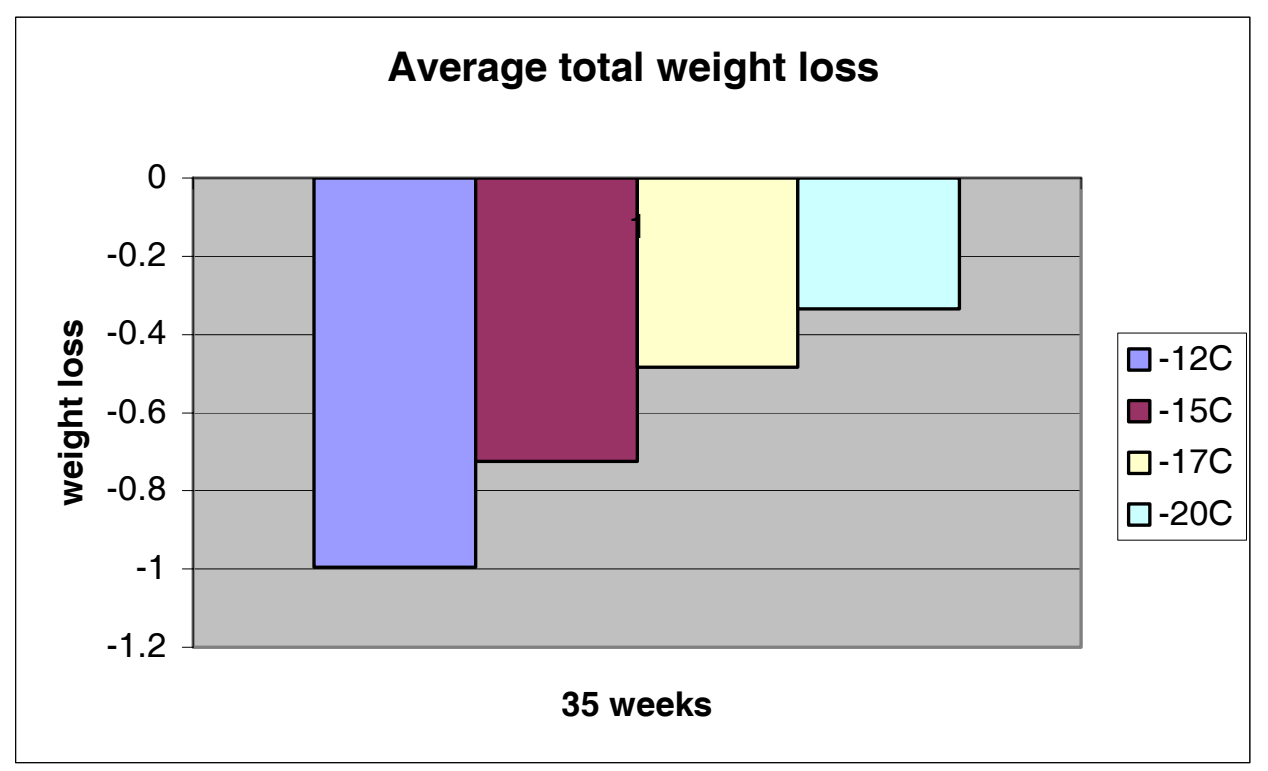

Figure 1

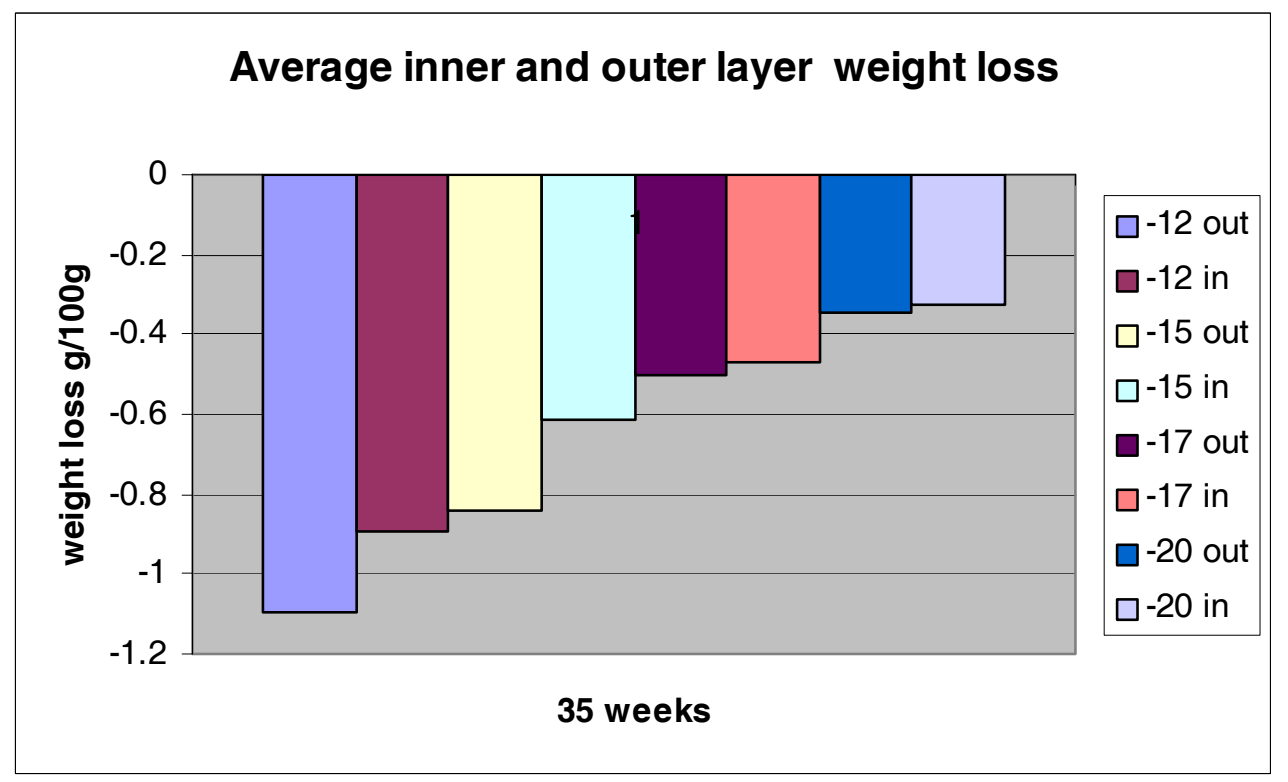

Figure 2 


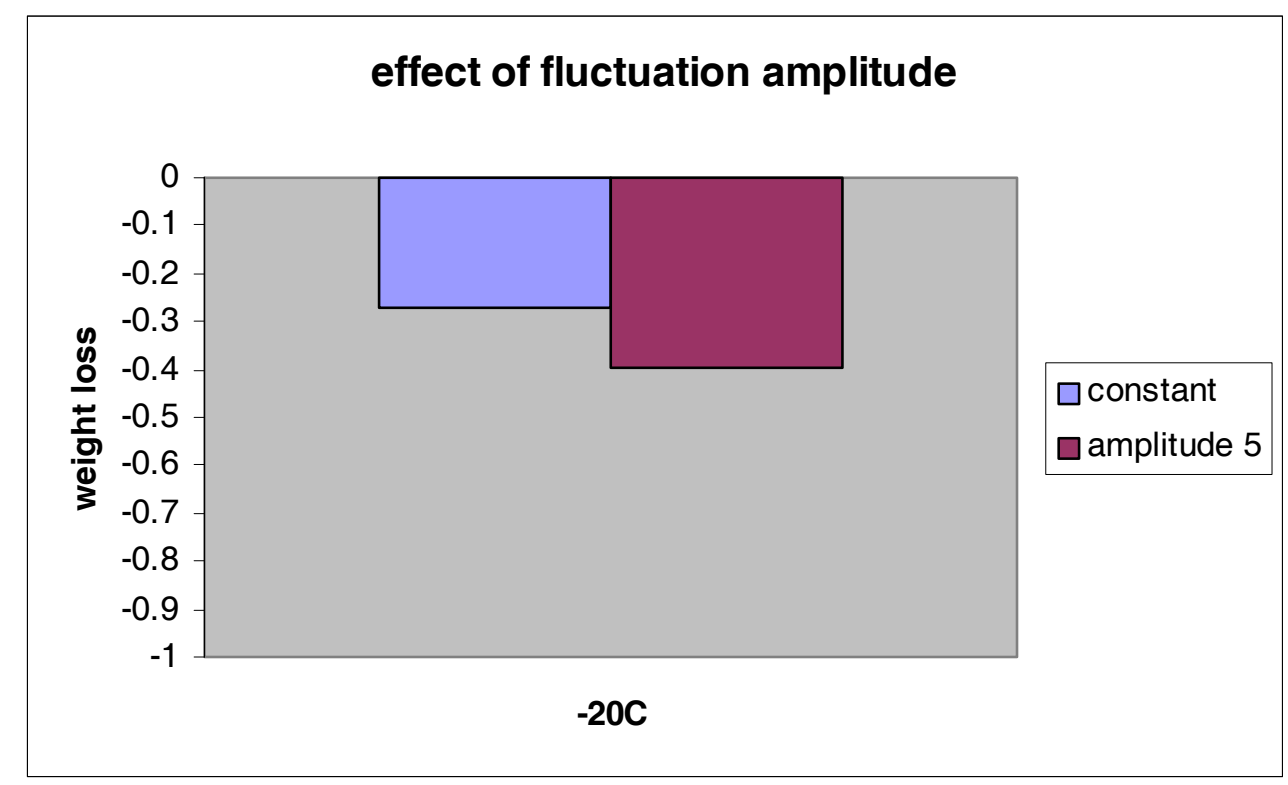

Figure 3

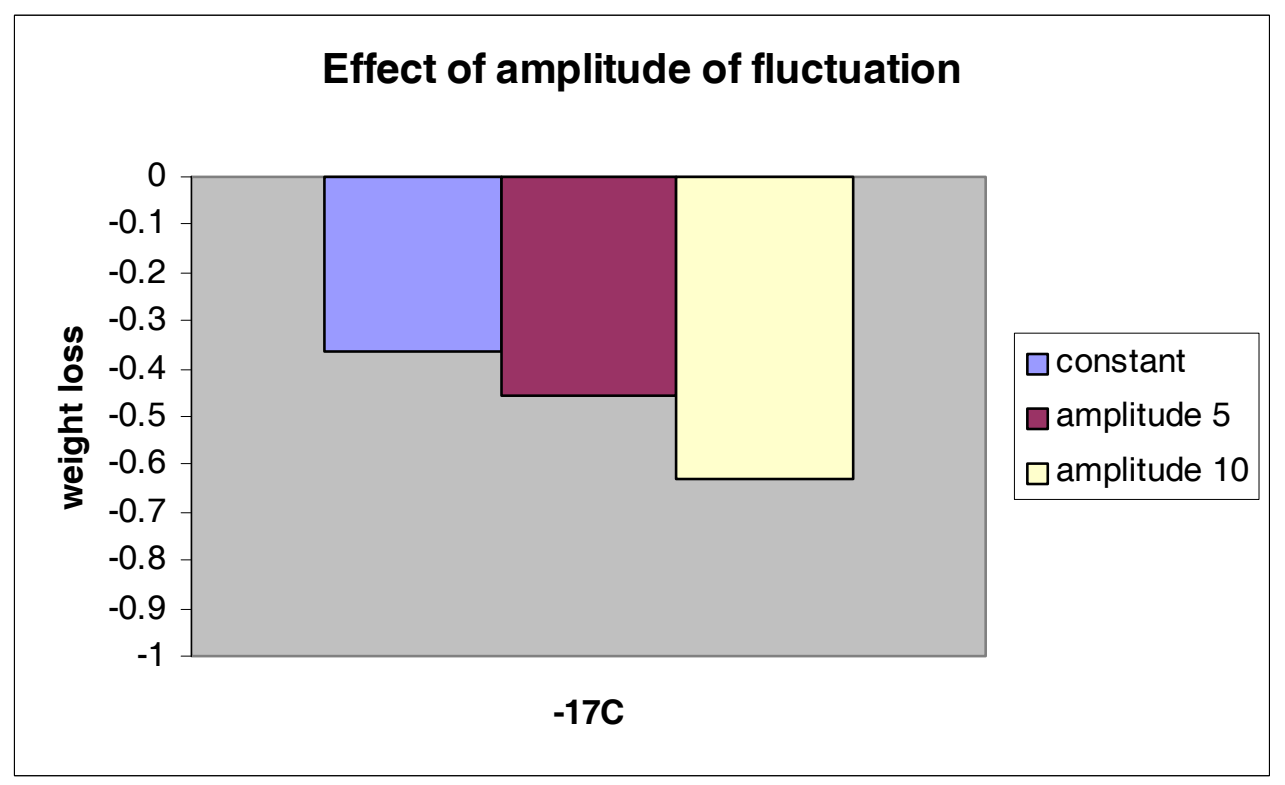

Figure 4 


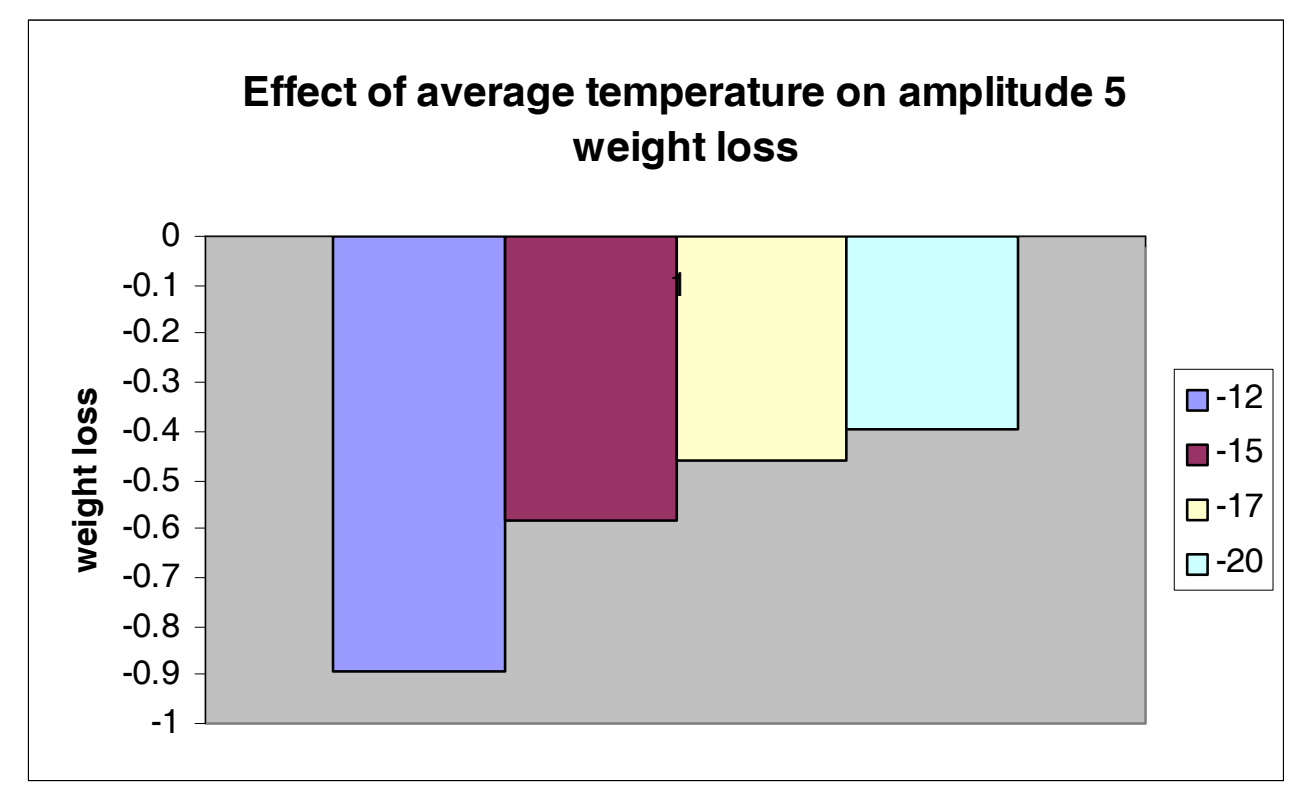

Figure 5

\section{Acknowledgement}

Support for this study was provided by the World Food Logistics Organization (WFLO)

\section{References}

Moleeratanond, W., A. Kramer, et al. (1979). "Effect of temperature fluctuation on energy consumption and quality changes of palletized foods in frozen storage." International Journal of Refrigeration 2(6): 221-8.

Van Arsdel, W. B., M. J. Copley, et al. (1969). Quality and Stability of Frozen Foods: Time-Temperature Tolerance and its Significance. 